\title{
THE STRUCTURE OF THE INTERSTELLAR MEDIUM AND STAR FORMATION IN THE MAGELLANIC CLOUDS
}

\author{
Michael A. Dopita \\ Mt. Stromlo and Siding Spring Observatories \\ The Australian National University \\ Private Bag, Woden P.O., ACT 2606 \\ Australia
}

\begin{abstract}
The Magellanic Clouds, by their proximity, offer a unique opportunity to study the detailed processes of star formation in a galactic context. This Review examines the dynamic and self-limiting feedback between star formation and the interstellar medium (ISM) in these galaxies. Energetic processes associated with star formation are responsible for pressurising the ISM, for maintaining the multi-phase structure of this medium and for ejecting copious quantities of both hot and cool gas to large heights above the galactic mid-plane. This circulation process determines the control of the star-formation rate.
\end{abstract}

\section{Introduction}

In order to understand the global structure and evolutionary characteristics of galaxies, a general theory of star formation is absolutely essential. Such a theory would also contain within it a model for the interstellar medium (ISM), since star formation rates ( SFR) are critically dependent on, and also control, the physical and chemical structure of this medium.

The Magellanic Clouds have a crucial rôle to play in the development of such a general theory of star-formation, since they may be studied for their integrated properties as galaxies in their own right, but yet are near enough that details of the individual star-forming complexes may be observed. Unfortunately, ground based observation tells us little about recent star formation in the low mass range (less than about 3 solar masses), and therefore this review will concentrate on the formation of stars more massive than about 10 solar masses which (Maeder 1981b) were formed within the last 20-30 Myr.

\section{Global Star Formation Rates and the Initial Mass Function.}

In principle, a complete sample of stars in the Hertzsprung-Russell (H-R) diagram, and a set of theoretical evolutionary tracks should suffice to give a direct determination of the Inital Mass Function (IMF) of massive stars. However, as emphasised in the review by Lequeux (1984), this approach is fraught with difficulties.

The catalogue material is complete only to around $\mathrm{B}=13 \mathrm{mag}$, which translates 
to about 15 solar masses on the zero age main sequence (Vangioni-Flam et al. 1980, Humphreys and McElroy, 1985). Apart from the selection effects, large errors are involved in the transformation between the observed and theoretical H-R diagrams.

The theoretical tracks on the H-R diagram are strongly affected by mass-loss from the massive stars (Chiosi, Nasi and Sreenivasan 1978, Maeder 1981b) and by convective overshooting (Bressan, Bertelli and Chiosi 1981). Neither the observed ratio of the yellow and red supergiants to the blue supergiants, nor the apparent spread in the observed main sequence agree with the theoretical models. However, the ratio of the blue to red supergiants, and the ratio of the Wolf-Rayet stars to the supergiant stars shows a systematic decline from the Galaxy to the LMC to the SMC. This is predicted by the radiatively-driven mass loss theory, in which the mass-loss rate depends critically on the metallicity of the atmosphere. The distribution of the supergiants in the H-R diagram suggests that these effects are more marked at higher stellar luminosities, again in agreement with radiation-driven mass-loss theories.

The IMF for massive stars is usually expressed as a power law; $N(m)=$ $\mathrm{am}^{-\mathrm{p}}$.dm, where the index, $\mathrm{p}$, lies in the range 1-3. Dennefeld and Tammann (1980) derived $\mathrm{p}=2.0$ for the LMC and $\mathrm{p}=1.4$ for the SMC. Humphreys and McElroy (1984) have shown that these results are strongly affected by incompleteness. Furthermore, the relative sparseness of the upper parts of the H-R diagram in the SMC combined with the greater distance modulus and its real scatter in the this quantity for this galaxy means that the mass range over which $\mathrm{p}$ can be determined is very restricted. When all these effects are taken into account, Humphreys and McElroy find no evidence for any dependence of IMF on galaxian environment ( $p=2.41,2.29$ and 2.14 for the Galaxy, LMC and SMC respectively.

The rate of star formation for massive stars can estimated either from the UV luminosity of these stars or from the luminosity of the associated HII regions. These methods are virtually equivalent, since, as emphasised by Lequeux (1980), the massive stars dominate the UV luminosity, and contribute essentially all the ionising photons. The H-Alpha luminosity of the HII region scales directly as the number of these ionising photons. We therefore expect a strong correlation between SFR, UV luminosity and $\mathrm{H}$-Alpha luminosity in any galaxy. A scatter in this correlation will be introduced by changing the slope of the $\mathrm{IMF}$, and by the effects of dustiness and/or age of individual HII complexes.

For the LMC Braunsfurth and Feitzinger (1983) find a such a correlation between the UV flux and the H-Alpha luminosity, though the quality of the photometry is probably responsible for the large scatter in the correlation. More quantitative data exists for the SMC, and Lequeux (1984) has derived the total number of ionising photons from the Schmidt (1972) H-Alpha photometry. An alternative approach was by Israel (1980) who used radio continuum flux measurements to derive the same quantity. For the SMC, the Lequeux and the Israel values are $4.6 \times 10^{51}$ and $1.3 \times 10^{51}$ Lyman Continuum photons per sec respectively, which gives some idea of the reliability of such estimates.

Vangioni-Flam et al. (1980) used the integrated UV flux measured with the D2B-Aura satellite (Maucherat-Joubert, Lequeux and Rocca-Volmerange, 1980) to derive the SFR in the Magellanic Clouds. A similar study using OAO-2 observations of a variety of galaxies has been carried through by Donas and Deharveng (1984). For the 
TABLE I : Estimated Current Global Rates of Star Formation

$$
\text { Galactic System } \mathrm{M}_{*}\left(\mathrm{M}_{\mathrm{o}} \cdot \mathrm{yr}^{-1}\right) \mathrm{M}_{\mathrm{T}} \mathrm{M}_{*}(\mathrm{Gyr}) \quad \mathrm{M}_{\mathrm{HI}} \mathrm{M}_{\mathrm{T}}
$$

\begin{tabular}{lcrl}
\hline Galaxy(local) & $3-10$ & $12-50$ & 0.04 \\
M33 & $0.2-0.5$ & $8-20$ & 0.12 \\
LMC & $0.3-0.8$ & $7-16$ & 0.09 \\
SMC & $0.05-0.16$ & $7-25$ & 0.24
\end{tabular}

References:

Berkhijsen(1982), Donas and Deharveng(1984), Israel (1980), Rocca-Volmerange, Lequeux and Maucherat-Joubert (1981), Smith, Biermann and Mezger (1978)

Magellanic Clouds, these and other results are summarised in Table I. Note that,although the galaxies listed in the Table vary over an order of magnitude in SFR and gas content, the specific rate of star formation (per unit mass of galaxy) is constant, within the errors. If we consider the relationship between SFR and gas content, then for the Sm and Irr galaxies the correlation is poor (Donas and Deharveng, 1984). The SMC, for example is very gas-rich and yet produces only $20 \%$ of stars per unit mass of gas that the LMC does.

\section{The Past History of Star Formation}

The colours of galaxies may be used to infer the past history of star formation. Tinsley (1972), Searle, Sargent and Bagnuolo (1973), Huchra (1977) and Larson and Tinsley (1978) have all shown that the position of a galaxy along the sequence in the (U-B) ; (B-V) diagram is sensitive to the average SFR in the past. On the other hand, the far UV fluxes are sensitive to the recent star formation rate. Rocca-Volmerange (1981) and Rocca-Volmerange, Lequeux and Maucherat-Joubert (1981) find that colour/coluor plots in the UV enable the ratio of present and integrated SFRs to be simply derived, the results are principally dependent on metallicity and on the IMF. Application of these results to the Magellanic Clouds ( Rocca-Volmerange, Lequeux and Maucherat-Joubert, 1981 ) and to the solar region of our Galaxy gives the following ratios of current SFR to average past SFR, assuming that the systems each have the same age:

LMC: $1.0-1.4, \quad$ SMC: $0.45-1.1, \quad$ Galaxy: 0.33 or less.

This implies that the mean rate of star formation in the Clouds has tended to remain more constant with time than has the Galactic SFR. However, this result should be treated with a certain amount of caution, since there is some fairly convincing evidence that the epoch of disk formation in the Clouds occured more recently than that of the Galaxy.

The average age of the field stars in both the LMC and the SMC has been derived by fitting theoretical isochrones to the Colour- Magnitude array. Since these arrays extend below the main sequence turn-off, the derived age is fairly accurate provided that the concept of a 'mean age' is appropriate for the Population. For the LMC there is clear evidence for a maximum is star-forming activity between 4 and $7 \mathrm{Gyr}$ ago 
(Butcher 1977, Stryker 1981, Stryker and Butcher 1981, Frogel and Blanco 1983). The SMC field stars in the outer parts of that galaxy appear to be even younger, with a mean age of 2-4 Gyr ( Hawkins and Bruck 1982, 1984). Given these shorter timescales,we may infer that the global SFR is in fact decreasing with time for the Galaxy, SMC and LMC. In view of the correlation between the gas content of these three galaxies and the mean age of their field stars, it is tempting to identify the past peak of star forming activity with the epoch of disk formation. This is discussed further below.

Since the present abundances in the ISM are the result of chemical enrichment from stars of all masses integrated over the history of the galaxy, a study of the ralative abundances of individual elements can furnish a great deal of information about the history of star forming processes. By combining the abundance data derived from HII regions, supernova remnants, Cepheids and supergiant stars, Dopita (1986) has been able to derive the abundances of many elements from helium through to the iron peak elements. These are given in Table II. The most striking characteristic of the data set is that the lighter elements, $\mathrm{C}, \mathrm{N}$ and $\mathrm{O}$ are more depleted in the Clouds than the heavier

TABLE II: Abundances by Number $(\log [\mathrm{H}]=12.00)$ and their Depletion Factors in the Magellanic Clouds

\begin{tabular}{lrrrrr}
\hline \hline Element & Solar & LMC & & SMC & \\
\hline 1. $\mathrm{H}$ & 12.00 & 12.00 & & 12.00 & \\
2. $\mathrm{He}$ & 11.00 & 10.96 & 0.04 & 10.93 & 0.07 \\
6. $\mathrm{C}$ & 8.62 & 7.76 & 0.86 & 7.16 & 1.46 \\
7. $\mathrm{N}$ & 7.93 & 7.13 & 0.80 & 6.50 & 1.43 \\
8. $\mathrm{O}$ & 8.84 & 8.32 & 0.52 & 8.09 & 0.75 \\
10. $\mathrm{Ne}$ & 7.99 & 7.48 & 0.51 & 7.30 & 0.69 \\
12. $\mathrm{Mg}$ & 7.60 & 7.20 & 0.40 & 6.97 & 0.63 \\
14. $\mathrm{Si}$ & 7.57 & 7.35 & 0.22 & 7.03 & 0.54 \\
16. $\mathrm{S}$ & 7.27 & 6.89 & 0.38 & 6.45 & 0.82 \\
17. $\mathrm{Cl}$ & 5.25 & 4.95 & 0.30 & 4.50 & 0.75 \\
18. $\mathrm{Ar}$ & 6.60 & 6.25 & 0.35 & 5.86 & 0.74 \\
20. $\mathrm{Ca}$ & 6.37 & 6.07 & 0.30 & 5.72 & 0.65 \\
21. Sc & 3.07 & 2.85 & 0.22 & 2.30 & 0.77 \\
22. $\mathrm{Ti}$ & 4.95 & 4.34 & 0.61 & 4.01 & 0.94 \\
24. $\mathrm{Cr}$ & 5.68 & 5.33 & 0.35 & 5.03 & 0.65 \\
26. $\mathrm{Fe}$ & 7.53 & 7.31 & 0.22 & 6.99 & 0.54 \\
& & & & & \\
\hline
\end{tabular}

ones such as $\mathrm{Si}, \mathrm{Mg}$ and $\mathrm{Fe}$. This is a strikingly different result from the results obtained from observation of metal- poor stars in our solar neighbourhood (Clegg, Lambert and Tomkin 1981). In these stars, a lower metallicity is associated with higher ( $\mathrm{O} / \mathrm{Fe})$ ratios, rather than the lower ( $\mathrm{O} / \mathrm{Fe}$ ) ratios seen in the Clouds.

The reason for this curious result can be sought in what we know about nucleosynthesis in stars of different mass ranges. Massive stars ( greater than about 12 solar masses ) are responsible for producing most of the $\mathrm{O}$ and $\mathrm{Ne}$, and much of the $\mathrm{Mg}$ 
and $\mathrm{Si}$ that finds its way back into the ISM. However, stars in this mass range are relatively inefficient in making S, Ar, Ca or Fe. (Weaver,Zimmerman and Woosley, 1978, Weaver and Woosley, 1980, Woosley and Weaver, 1982). These elements are made much more effectively in carbon deflagration supernovae of intermediate mass stars. (Sugimoto and Nomoto 1980, Nomoto 1984a,b). Wood, Bessell and Fox (1983),

have identified the precursors of such stars in the Clouds, which exist as long period Asymptotic Giant Branch variables with pulsation masses in the range $3.5-7$ solar masses. These stars have core masses right up to the Chandrasekhar limit, and must have evolved from stars of up to 7.8 to 9.2 solar masses, depending on the efficiency of mass loss. When the abundance of these stars, relative to the massive stars, is taken into account, it is evident that they are very important contributors to the enrichment of the ISM.

The difference between the local Galactic abundances at earlier times and the present - day abundances in the ISM of the LMC and SMC can therefore be understood if, in the early Galaxy, there existed a violent burst of star formation with a greater production efficiency of massive stars (i.e, a flatter IMF). The figures in the second column of Table I and the fact that the initial chemical enrichment of the Galaxy was relatively prompt together strongly suggest that this flattening of the IMF was a result of a very high specific rate of star formation, heating of the ISM and therefore a high Jeans Mass in the gas of the newly collapsed Galactic disk.

Finally, the chemical enrichment data is consistent with the greater youth of the galactic disks of the Clouds. Provided that the disk is old enough, stars of low mass are believed to be responsible for much of the $\mathrm{C}$ and $\mathrm{N}$ enrichment of the ISM via mass-loss and planetary nebula ejection (Peimbert and Torres-Peimbert, 1971; Kaler, 1974). Enrichment of the outer layers of such stars occurs through various convective dredge-up episodes (Becker and Iben, 1979,1980; Renzini and Voli 1981). Stars with masses below about times 3 solar become Carbon stars. Despite the relatively high present-day abundance of these stars in the LMC, they can have had little effect on the enrichment of either the SMC or the LMC. Not only is the Carbon abundance in the gas-phase ISM very low in these systems, but also the $2200 \AA$ absorption feature (generally thought to be due to graphite grains) is very weak in the LMC and absent in the SMC along nearly all sight-lines (e.g. Koorneef and Code, 1981; Bromage and Nandy, 1983; Fitzpatrick and Savage, 1984).

\section{The Distribution of Recent Bursts of Star-Forming Activity.}

The proximity of the Clouds means that, even with telescopes of modest aperture, the ages of individual supergiants and young star clusters can be determined with a high degree of precision. This enables us to both define the regions of star-formation and to study their recent temporal evolution. Thus, theories of star-formation can be subjected to observational scutiny. (See also the contribution by Feitzinger, this volume.)

In many respects the two Magellanic Clouds are strikingly different. The SMC shows considerable evidence for severe, possibly fatal, tidal disruption which results from its close passage to the LMC. The LMC, on the other hand, is still well organised into a disk, and star-formation is more typical of a normal galaxy. 


\section{1. $\mathrm{SMC}$}

The structural features of star-forming regions over the past $10^{8}$ years or so can be inferred from the spatial distribution of the Supergiants and the Cepheids. For the SMC supergiants, the definitive study remains that of Azzopardi and Vigneau (1977), who used catalogue material by Sanduleak $(1968,1969 a)$ and Azzopardi andVigneau (1975). The distribution of the SMC Cepheids was discussed by Hindman (1967) and Sanduleak (1969b), and the distribution of the blue and open clusters by Bruck (1975).

From these studies, and the earlier work of de Vaucouleurs (1955), it is apparent that the recent star formation is concentrated to the noth and the east of the older stellar bar population (Azzopardi and Vigneau's Bc region). A second concentration of young stars is to the east and south east in the Inner Wing ( $\mathrm{Aa}$ and $\mathrm{Ab}$ region) and the outer Shapley Wing (R.A. > $1 \mathrm{hr} 35 \mathrm{~min}$ ) extending towards the LMC. This outer Wing region consists mainly of young blue stars with an average age of order $10 \mathrm{Myr}$ ( Westerlund, 1964a), and is associated with the only supergiant HII shell in the SMC (Meaburn,1980).

These two regions associated with a high SFR in recent times are apparently associated with two distinct, gas-rich tidal features or arms, produced by the recent disruptive tidal encounter of the SMC with the LMC (Mathewson and Ford,1984; Murai and Fujimoto 1980; Fujimoto and Murai, 1984). Star formation in these regions must have commenced within the last $(1-2) .10^{8}$ years, since little or no sign of these features appears in either the spatial distribution or the kinematics of the SMC planetary nebula population (Dopita et al. 198 ). This timescale is consistent with the collision timescale between the two Clouds. Distance moduli derived from both supergiants (Azzopardi, 1982 ) and from Cepheid variables (Mathewson, Ford and Visnanathan, 1985 ) show that the SMC has a considerable spatial extension, up to about $10 \mathrm{Kpc}$, in depth. The NE extension is associated with a $\mathrm{HI}$ arm-like feature on the far side of the SMC, which may be identified with a tidal counter-arm (Dopita et al. 1985). The SMC is thus an ideal laboratory for study of the processes of shock-induced star-formation. However, such a study would require a more detailed understanding of spatial correlations between $\mathrm{HI}$ gas dynamics and current SFRs than we possess at present.

\section{2. $\mathrm{LMC}$}

The overall structure of the LMC is much better organised than the SMC. The older pooulations are located in the Bar and in a flat disk (Freeman, Illingworth and Oemler, 1983), whereas the younger stars tend to occur in well-defined super-associations such as the Shapley Constellations or 30 Doradus (McKibben Nail and Shapley, 1953; Westerlund and Smith, 1964; Westerlund and Mathewson, 1966 ). Whether or not spiral structure is present has been a point of contention, although studies of spiral tracers such as HI, HII regions, dust globules, supergiants, Cepheids and Carbon stars tends to support the idea that some incipient spiral structure does exist. (Payne-Gaposhkin, 1972; de Vaucouleurs and Freeman, 1973; Martin et al., 1976; Schmidt-Kaler, 1977). The mathematical methods of pattern rcognition have been applied to the young populations of the LMC (Feitzinger and Braunsfurth, 1984 and Feitzinger, this conference). This work emphasises the dangers of using visual inspection for pattern recognition, but nevertheless shows that real correlations do exist at a variety of spatial scales. 
The structure of the eastern boundary of the LMC is confused, and the $21 \mathrm{~cm}$ line profiles show an extensive region of splitting, combined with a local perturbation of the velocity field (Rohlfs et al.,1984). This feature may be associated with the ram-interaction between the disk gas and the hot halo of our galaxy (Meatheringham and Dopita, 1986). The LMC is moving through this medium at a velocity of order $400 \mathrm{~km} \cdot \mathrm{s}^{-1}$, and this should be sufficient to strip some of the HI out of the galaxy, and by shock interactions,enhance star formation in this portion of the LMC. This shocked boundary may be defined by a dust lane and by HII regions including N158c, N159 and N160 where IR protostars have been identified (Gatley et al. 1981; Epchtein et al. 1984 ).

Here we will concentrate on the so-called supergiant loops or shells of ionised and neutral gas which together cover about ten percent of the area of the disk of the LMC. These have been identified and mapped from very deep H-Alpha plates by Goudis and Meaburn $(1978)$ and Meaburn $(1980,1981)$. On the basis of their size
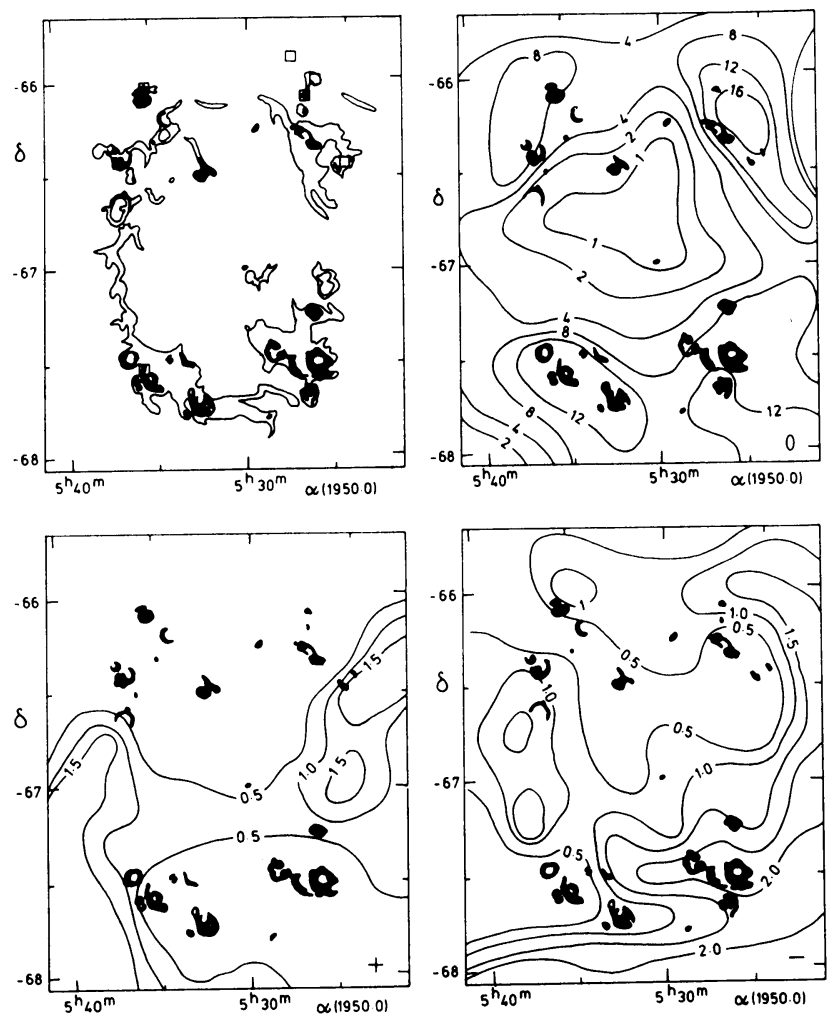

Figure 1. The HI structure of the Shapley III/ LMC4 supergiant loop (Dopita, Mathewson and Ford,1985). Top left to bottom right; the distribution of HII regions, the distribution of HI in the plane of the LMC, the high velocity approaching $\mathrm{HI}$ component and the high velocity receding shell. Contour units are $10^{20} \mathrm{H}$ atoms $\mathrm{cm}^{-2}$. 
distribution alone, these form a population which is distinct from the 'normal' HII regions. In all cases, they contain at least one Lucke and Hodge (1970) clustersomewhere near the center and a field population of young blue stars which is prominent in the far UV atlas of Page and Carruthers (1981). Most of the supergiant shells (LMC 2,3,4,7 and 8) are associated with dense rings or loops of HI, which contain in the region of $(0.5-2.5) \times 10^{7}$ solar masses of gas. This argues for a compressive process in the gas.

The stellar ages derived by Isserstedt (1984) enables us to study the temporal evolution of star-forming activity in these regions. In all cases, the stars near the center are, on average, older than those on the periphery. In the case of LMC4, associated with Shapley III, this progression is well defined, and shows that the region of star formation has spread outwards at about $35 \mathrm{~km} . \mathrm{s}^{-1}$ over the past $14 \mathrm{Myr}$, although at any one epoch, star formation is active over a range of different radii.

The detailed dynamics of LMC 2 has been studied by Caulet et al. (1982), and the Shapley III region has been investigated by Dopita, Mathewson and Ford (1985). In the case of LMC 2, located to the east of 30 Dor near $5 \mathrm{hr} 41.5 \mathrm{~min} ;-69^{\circ} 25$, both ionised gas and the $\mathrm{HI}$ are ballooning out of the far side of the galactic plane. About $5 \times 10^{6}$ solar masses of gas are involved with an expansion velocity of about $30 \mathrm{~km} . \mathrm{s}^{-1}$. The parameters of this shell are comparable to the largest of the Heiles (1979) HI shells in our own Galaxy. Caulet et al. (1982) suggest that LMC2 could result from the combined effect of stellar winds and supernovae.

The supergiant loop LMC 4 forms an almost perfect circle in the plane of the LMC almost $2 \mathrm{kpc}$ in diameter, centered at $5 \mathrm{hr} 31 \mathrm{~min} ;-66^{\circ} 54$. The centre of this shell is almost entirely devoid of HI at the velocity of the disk. That is to say that it has been swept almost clean of gas, although this region was an active site of star-formation only 10 Myr ago or so. Much of this gas has been swept into the shell, but at least $2.5 \times 10^{6}$ solar masses of HI has been flung out of the plane of the LMC with a velocity of about $35 \mathrm{~km} \cdot \mathrm{s}^{-1}$ (see figure 1). These data imply an age of about $15 \mathrm{Myr}$ and an energy of about $10^{53}$ ergs. Dopita, Mathewson and Ford (1985) estimate, from studies of the stellar content and the associated supernova remnants, that the energetic processes associated with the young stars are sufficient to drive the spectacular dynamics of this region.

Both LMC2 and LMC4 show graphically that stochastic self-propagating star formation (SSPSF) (Mueller and Arnett, 1976; Gerola and Seiden, 1978; Seiden and Gerola, 1979) does indeed occur in real galaxies. These models have been specifically applied to the LMC ( Feitzinger et al. 1981). The low velocity shear that exists in this galaxy results in partly ordered structures that bear a morphological resemblance to the Giant Loop complexes. The observations of Dopita, Mathewson and Ford (1985), allow us to infer the physical processes that control this mode of star-formation. However, since these depend so intimately on the structure of the ISM, let us first briefly review the properties of this medium.

\section{The Three-Phase Interstellar Medium in the Magellanic Clouds.}

In the solar neighbourhood, it is apparent that the energetic processes associated with the young stellar population control the structural properties of the ISM (Cox and Smith, 1974; McKee and Ostriker, 1977; McCray and Snow, 1979; Cox, 
$1979,1981)$. Locally, a low - density phase at coronal temperatures appears to occupy a large fraction of the volume and provide a background pressure with which the other phases of the ISM (the warm, atomic and cool, molecular component) are in quasi steady equilibrium.

There is some evidence from the properties of supernova remnants and H II regions (Dopita, 1979; Tuohy et al. 1982; Long, Dopita and Tuohy, 1982; Long, 1983; Wilson, 1983 ) that both a cloud and intercloud medium exist in the Clouds. The cloud medium has a density in the range $10-30 \mathrm{~cm}^{-3}$ and a probable temperature near $100 \mathrm{~K}$, whereas the intercloud density is only $0.1-0.3 \mathrm{~cm}^{-3}$ and the temperature is maintained near $10^{4} \mathrm{~K}$, so that the two components are in approximate pressure equilibrium with one another with $\log [\mathrm{P} / \mathrm{k}]=3.1 \pm 0.4 \mathrm{~cm}^{-3} . \mathrm{K}$.

A source of energy input is required to maintain the intercloud medium, since isits cooling timescale is only about $2 \times 10^{7}$ years at LMC abundances. If it is confined within one scale height of the plane, this is shorter than the sound-crossing timescale. In models of the Local ISM, this is maintained by electron conduction from the coronal component (McKee and Ostriker, 1977) or by direct mechanical heating from supernova explosions (Cox, 1981). However, the solar neighbourhood may be exceptional, since we are surrounded by sites of active star-formation. An alternative, and more ubiquitous form of heating is by soft X-ray photoionisation heating. From surveys of the soft X-ray flux in the LMC and SMC (Long, 1976; Long, Helfand and Grabelsky, 1981; Inoue, Koyama and Tanaka, 1983); $\mathrm{N}_{\mathrm{x}}=(5-8) \times 10^{46} \mathrm{~s}^{-1}$ for the SMC and $N_{x}=(3-5) \times 10^{47} \mathrm{~s}^{-1}$ for the LMC. Thus mean omnidirectional photon fluxes of order $10^{3} \mathrm{~cm}^{-2} \mathrm{~s}^{-1}$ may be maintained in the intercloud disk gas. Such fluxes are not only capable of maintaining a warm intercloud medium, but indeed, this is favoured at Magellanic Cloud abundances. A theoretical calculation of the structure of the ionised region that can be maintained by this surface flux in the interclour medium has been carried through by Dopita (1985). At LMC abundances, the temperature is maintained at about $10^{4} \mathrm{~K}$ to a column density of order $10^{21} \mathrm{~cm}^{-2}$, which corresponds to a distance of order $1 \mathrm{kpc}$ in the intercloud medium. The temperature falls sharply when the degree of ionisation falls below about 0.03 . At SMC abundances the warm intercloud can be maintained out to about $3 \times 10^{21} \mathrm{~cm}^{-2}$, whereas at Solar abundance, the warm intercloud exists only to a column density of $2 \times 10^{20} \mathrm{~cm}^{-2}$.

The coronal component of the ISM certainly requires direct heating by supernova shocks for its continuing existence. However, the supernova rate in the LMC of about 1 per 200 years is not sufficient to maintain this component with a large filling factor throughout the disk medium. The regions where the coronal component dominates can be identified with regions in which star formation is much higher than the mean, i.e. the Supergiant loops which together occupy about 10 percent of the total disk area. Here, as already mentioned, the HI has largely been stripped out, leaving the ejecta of supernova explosions an almost unhindered passage to the halo of the LMC. In this way we expect the coronal gas to bubble out of the disk to fill out a very extensive hot halo, which supplies a confining pressure to the disk gas.

Direct evidence for the existence of such a halo comes from resonance line absorption observations of highly ionised species against Magellanic Cloud O and B stars. Using the IUE satellite, absorption due to C IV, Si IV and, in some cases N V has been detected over a wide range of velocities ( Savage and de Boer, 1979; de Boer, Koorneef and Savage 1980; Gondhalekar et al. 1980; de Boer and Nash 1982; 
Fitzpatrick and Savage 1983 ). The dynamics of this gas are consistent with a extensive sheet of absorbing material which is rising into the halo at a velocity of order 50 $\mathrm{km} . \mathrm{s}-1$. Estimates based on the energy balance of this gas as it leaves the plane suggest an initial temperature approching a million degrees. Thus, the absorption seen with IUE does not arise in this gas, but rather, far out in the halo as the gas cools. Since the column density seen in absorption is dependent on number of absorbers in the non-ionisation equilibrium cooling zone between roughly $10^{5}$ and $10^{4} \mathrm{~K}$, cooling calculations can be used in conjunction with the absorption and velocity data to derive very stringent limits on the physical conditions in the halo gas. Songaila (1981) was the first to attempt this but was unable to obtain consistent results between the different absorbing species. Dopita (1985) has carried though new non-equilibrium isochoric cooling calculations with Galactic and Magellanic Cloud abundances and finds that a much better agreement can be obtained. The different ions now give results that agree within a factor of two or so. From these calculations, and the energy input estimates based on supernova rates, the (approximate) halo parameters given in table 3 result.

TABLE III: Estimates of the (Hot) Halo Parameters of the Galaxy and the Magellanic Clouds

\begin{tabular}{llll}
\hline Halo Parameter & Galaxy & LMC & SMC \\
\hline $\begin{array}{l}\text { Mass Flux } \\
\left(M_{0} \cdot{ }^{-1}\right)\end{array}$ & $5-10$ & $0.4-1.0$ & $0.1-0.3$ \\
$\begin{array}{l}\text { Log Initial Pressure } \\
\left(\mathrm{cm}^{-3} . \mathrm{K}\right)\end{array}$ & $3-3.5$ & $3.0-3.5$ & $3.0-3.5$ \\
$\begin{array}{l}\text { Log Initial Temp. } \\
(\mathrm{K})\end{array}$ & $5.7-6.1$ & $5.7-6.1$ & $5.7-6.1$ \\
$\begin{array}{l}\text { Log Radiated Power } \\
\left(\mathrm{erg} . \mathrm{s}^{-1}\right)\end{array}$ & $40.8-41.1$ & $39.5-39.8$ & $39.0-39.3$ \\
$\begin{array}{l}\text { Log Cooling Time } \\
(\mathrm{yr})\end{array}$ & $7.4-8.0$ & $7.8-8.4$ & $8.0-8.6$ \\
$\begin{array}{l}\text {-Log Mean Density } \\
\left(\mathrm{cm}^{-3}\right)\end{array}$ & $3.3-2.7$ & $3.5-3.1$ & $3.5-3.1$ \\
\hline
\end{tabular}

The regions where hot gas is being fed into the halo are characterised by large local overpressures. In the Shapley III / LMC4 region, for example, Dopita, Mathewson and Ford (1985) estimate that the pressure in the intercloud may exceed the mean by about a factor of 10 . Pressure gradients set up bu bursts of star formation therefore encourage flow into the halo. Since the halo cooling timescales (Table 3) are so long compared with the sound crossing time between adjacent sites of star formation, the halo gas as a whole can even out these pressure fluctuations. The halo component therefore plays a vital rôle in pressurising the disk gas between episodes of star formation, and in moderating the fluctuations in this pressure induced by episodes of star formation. 


\section{Towards a Theory of Star Formation}

In the past, an analytic approximation to a law of star-formation which has received particular emphasis is that propsed by Schmidt (1959) and which states that the number of stars formed in a unit volume per unit time is proportional to some power, $P$, of the local density, where $P$ is about 2 . This law was given some theoretical justification from the fragmentation theory of Field and Saslaw (1965).

Sanduleak (1969b) was the first to propose that, in a thin disk, the surface density of stars or gas could stand in place of the volume densities, and this has been the basis of the observational tests of the validity of the law. In the SMC, provided that luminous OB stars, Cepheid variables or HII regions are used to trace the regions of star formation, a Schmidt - type law appears to work quite well with $P$ between 1.6 and 2.6 (Sanduleak, 1969b; van Genderen, 1969; Hamajima and Tosa 1975 and Azzopardi and Vigneau, 1977 ). However the correlation does not extend to faint stars, presumably because their greater average age permits the parent gas clouds to disperse. In the LMC, the OB stars correlate much less well with the gas (Martin et al. 1976). However, the youngest populations (HII regions or WR stars) show the best correlation with P between 1.6 and 1.9 (Tosa and Hamajima 1975; Martin et al. 1976).

Apart from the self-evident truth that stars are born in clouds of gas, what is the physical content of the Schmidt law in the context of the Magellanic Clouds? In the previous section we showed how the various phases of the ISM co-exist approximately in pressure balance. In regions of active star formation, the energetic processes of mass loss and supernova explosions are sufficient to produce a large local overpressure. The evidence from the Shapley III area strongly suggests that this overpressure drives an isothermal shock into the intercloud gas, so producing the $\mathrm{HI}$ ring seen in figure 1 . The fact that star formation is currently occuring in a whole set of HII regions and HII complexes around the periphery suggests that this isothermal shock triggers the collapse of dense, pre-existing self-gravitating cloudlets. If these are approximated as isothermal gas spheres, their density at the surface will be related to the mean pressure $<\mathrm{P}>$ in the ISM:

$$
<\mathrm{P}>=\rho \mathrm{c}^{2}
$$

where $\mathrm{c}$ is the speed of sound in the cloud material. Since the clouds are assumed isothermal, their density profile follows a law of the form $p(r)=\mathrm{Ar}^{-2}$, so that the mean surface density of matter in each cloud is:

$$
\sigma=M / \pi R^{2}
$$

where the cloud mass $M$ and the cloud radius $R$ are related to the density at the surface of each cloud by:

$$
p=M / \pi R^{3}
$$

Now, in a cloud which has been tipped into instability by an increased external pressure $P(t)$, the ram pressure of infall is of the same order as the external pressure:

$$
\mathrm{P}(\mathrm{t}) \approx \rho \mathrm{v}_{\mathrm{ff}}{ }^{2}=2 \rho \mathrm{GM} / \mathrm{R}
$$


Hence, from equations 6.3 and 6.4;

$$
\mathrm{P}(\mathrm{t}) \approx 2 \mathrm{GM}^{2} / \pi \mathrm{R}^{4}
$$

However, Schmidt's Law with an index $P=2$ implies that the number of stars produced in the collapse $\mathrm{N}_{*}$ is ( from equation 6.2 ) given by:

$$
\mathrm{N} *=\alpha \sigma^{2}=\alpha \mathrm{M}^{2} / \pi \mathrm{R}^{4}
$$

where $\alpha$ is a constant of proportionality. Since 6.5 and 6.6 have the same functional dependence on the cloud parameters, this shows that Schmidts law with an index of two is equivalent to saying that the number of stars wrung out of any cloud is proportional to the overpressure! In LMC2 and LMC4, this overpressure can be estimated from the velocity of propagation of the isothermal shock or the rate of spreading of the star-forming area, and lies in the range $(2-10) \times 10^{-12}$ dynes. $\mathrm{cm}^{-2}$, about 10-20 times the pressure in the undisturbed ISM. The remarks in the previous section should have made clear that this overpressure is self-limiting because it can be relieved into the halo.Since the overpressure is proportional to the number of young stars per unit volume and in turn governs the rate of star formation, the region of star-forming activity expands uniformly until the supply of $\mathrm{HI}$ clouds is exhausted.

The physical process whereby the stars are wrung out of the HI clouds is most likely the operation of the gravitational instability in the outer portion of the cloud which has suffered the isothermal shock. Elmegreen and Elmegreen (1978) have shown that a plane-parallel layer will become unstable to its self-gravity when the surface density in the shocked layer, $\mathrm{s}$, exceeds

$$
\mathrm{s}>0.91[\mathrm{P} / \pi \mathrm{G}]^{1 / 2}{\mathrm{gm} . \mathrm{cm}^{-2}}^{-2}
$$

With typical cloud parameters,temperature $50 \mathrm{~K}$, surface density $12-60 \mathrm{~cm}^{-2}$, this condition will be satisfied for all HI clouds with a mass greater than about a few thousand solar masses. The time required to build up the critical surface density in the shocked layer depends on the cloud density, but is typically about $1 \mathrm{Myr}$. Clouds which were initially very massive may undergo more than one star-forming episode. These two factors are sufficient to explain why the layer which is currently making stars in LMC4 is relatively thick, and why the HI ring (which, in this picture, delineates the intercloud shock) is larger than the ring of bright HII regions (which delineate the sites of recent star formation).

This picture of self-propagating star formation differs in several respects from the picture developed by Elmegreen and Lada (1977), Elmegreen and Elmegreen (1978) or Bruhweiler et al. (1980). It is on a grander scale, and limits itself by stripping out the $\mathrm{HI}$ and throwing it up and out of the plane as both hot and cool or cold gas.

Similar ideas are found in models of stochastic self-propagating star formation (SSPSF) (Mueller and Arnett, 1976; Gerola and Seiden, 1978; Seiden and Gerola, 1979), which attempt to address the question of the galaxy-wide distribution of star forming regions. They are characterised by a probability that star formation in a given spatial cell will initiate star formation in an adjacent shell,by a mean stellar age, and also by a diffusion timescale for the return of gas to a cell in which star formation has been triggered. This diffusion process makes the star formation self-limiting. Such models 
are capable of explaining many of the structural features of spiral galaxies. In particular, for low mass systems, star formation is expected to become quite sporadic as the system as a whole begins to behave a little like a relaxation oscillator (Gerola, Seiden and Schuman 1980).

The SSPSF model has been specifically applied to the LMC (Feitzinger et al. 1981 , Feitzinger, this volume). In this galaxy, the low velocity shear results in structures that are partly ordered in a manner similar to that which is observed. However, the propagation velocity of the star-forming region is lower (about 10 $\mathrm{km} . \mathrm{s}^{-1}$ ), than the $35 \mathrm{~km} . \mathrm{s}^{-1}$ which is observed. As discussed above, this propagation velocity depends on the cloud/intercloud structure of the ISM and is also a measure of the overpressure produced by the local star-formation. These factors could be included in an improved version of SSPSF.

What of the material thrown into the Halo? This material undergoes 'storage' for a timescale that depends if it was thrown out as warm or cool gas at about $35 \mathrm{~km} . \mathrm{s}^{-1}$ or if it was expelled at coronal temperatures. The cooler gas, assuming that it moves ballistically, will return to the plane in about $1-2 \times 10^{8}$ years. The Coronal gas will first cool, and the cooled filaments will then move approximately ballistically. The Halo residence timescale for the Coronal medium may therefore be as long as $10^{9}$ years. Thus, using the figures of Table 3 , and the measued HI mass flux in the vicinity of the supergiant loops, we estimate that $2-6 \times 10^{8}$ solar masses of gas are stored in the halo of the LMC. As this material falls back to the plane, its kinetic energy is dissipated in cloud-cloud collisions, at the rate of $0.5-5 \times 10^{39} \mathrm{erg} . \mathrm{s}^{-1}$, shared equally between the cooled halo material and the ejected cool gas.

The returning gas cloudlets will collide with the $\mathrm{HI}$ clouds in the plane of the LMC. The resulting shocks may well induce local star-formation, but the major effects of these collisions are to increase, or rather, maintain, the z-velocity dispersion of the $\mathrm{HI}$ and to modify the size spectrum of the HI clouds. Without this source of energy and momentum input, the HI clouds in the plane would gradually coalesce and settle into a very thin disk. The infalling cloudlets, on the other hand, shatter the HI clouds and stir them up to maintain the $15 \mathrm{~km} . \mathrm{s}^{-1}$ or so velocity dispersion which characterises the HI $21 \mathrm{~cm}$ line profiles and the young stellar clusters (Freeman, Illingworth and Oemler, 1983). The cloud stize spectrum of the ISM is therefore maintained by the dynamic balance between cloud shattering and cloud coalescent collisions.

Dopita (1985) has used these ideas to develop a simple parameterisation of global star-formation rates in disk galaxies. The basis of this is that the mean pressure in the ISM, which, as shown above, may be identified with the pressure in the halo, is maintained by energetic processes associated with both the young and old stellar populations. These processes are also responsible for maintaining the z-velocity dispersion of the gas, so that there is an equipartition between the turbulent and thermal pressure terms. The first of these assumptions can be written as:

$$
\mathbf{P}=\alpha \partial \mathrm{m}_{*} / \partial \mathrm{t}+\beta \mathrm{m}_{*}
$$

where the first term is the pressure contribution from young OB stars in the form of stellar winds and Type II Supernovae, and the second term reflects the energy input, per unit volume and time, of the older stellar population. The source of energy input in this case is predominantly through Type I Supernovae.The second assumption gives: 


$$
P=f_{1}\left\langle p_{g} v_{z}^{2}>\right.
$$

where $f_{1}$ is a factor of order unity, and $p_{g}$ is the gas density in the plane. These two can be related if we adopt the disk model of van der Kruit and Searle (1981). In this, the z-density distribution of matter (gas and stars) has the form:

$$
p(z)=p(0) \cdot \operatorname{sech}^{2}\left(z / z_{0}\right)
$$

and the rms z-velocity dispersion is related to the total surface density of matter, $\sigma_{\mathrm{T}}$, and to the scale height of matter, $\mathrm{z}_{\mathrm{O}}$, by:

$$
\left\langle\mathrm{v}_{\mathrm{z}}{ }^{2}>^{1 / 2}=\left(\pi \mathrm{G} \sigma \sigma_{\mathrm{T}} \mathrm{z}_{\mathrm{o}}\right)^{1 / 2}\right.
$$

From $6.8,6.9$ and 6.11 in the limit where only the young stars pressurise the interstellar medium, we have:

$$
\begin{aligned}
\partial \mathrm{M}_{*} / \partial \mathrm{t} & =0.5 \pi \mathrm{G} \alpha^{-1} \mathrm{f}_{1} \mathrm{~V} \sigma \sigma^{\sigma} \mathrm{T} \\
& =\pi \alpha^{-1} \mathrm{f}_{1} \mathrm{z}_{0} \mathrm{M}_{\mathrm{T}}^{\sigma_{\mathrm{g}}} \mathrm{g}
\end{aligned}
$$

where $\mathrm{V}$ is the total volume available for star formation and $\mathrm{M}_{\mathrm{T}}$ is the total mass of the galaxy. Thus, in gas rich systems the star formation rate should scale as the product of

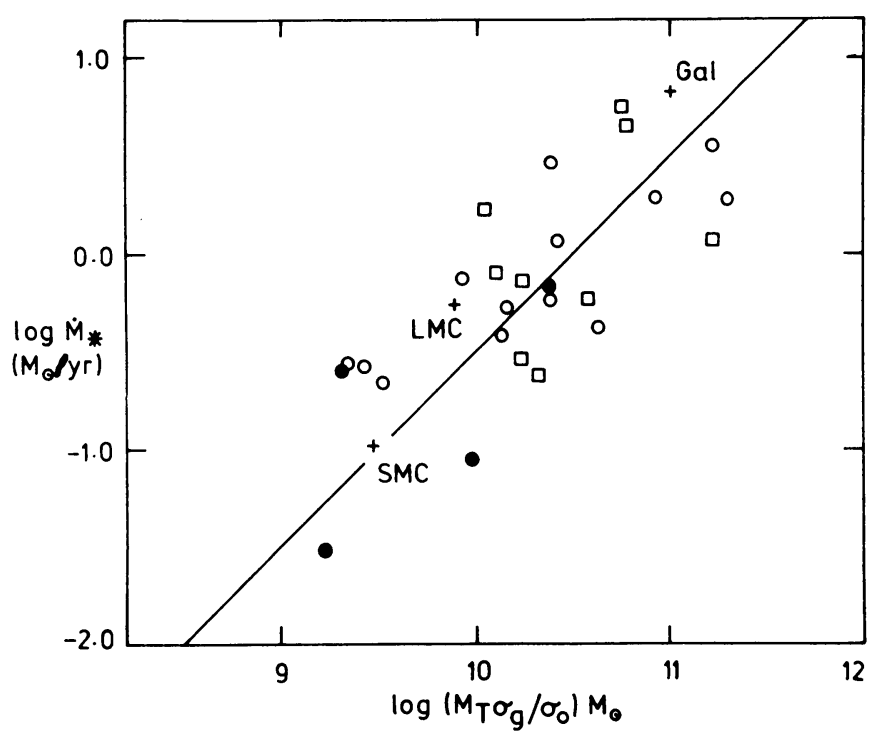

Figure 2: The rate of star formation as a function of the product of total mass of the galaxy and mean surface density of gas, for the gas-rich galaxies in the sample of Donas and Deharveng (1984). The normalising density is $3 \times 10^{-3}$ g.cm ${ }^{-2}$. The line is a best fit, assuming that eqn. (6.12) applies. Irr and Sm galaxies are shown as filled circles, $\mathrm{Sc}$ and $\mathrm{Sd}$ galaxies as open circles and $\mathrm{Sb}$ galaxies as open squares. 
total mass and mean surface density of gas. This is indeed quite a good approximation, as shown in Figure 2.

When the pressure term resulting from the old stellar population is included, we obtain a differential equaion on the gas content:

$$
\alpha f_{2}{ }^{-1} . \partial \sigma_{g} / \partial t+\sigma_{g}\left(\pi f_{1} G \sigma_{T} z_{0}+\beta\right)-\beta \sigma_{T}=0 \quad 6.13
$$

where $f_{2}$ is the fraction of gas retained in the stellar population in each generation of stars. Its value is about 0.7. Equation (6.13) predicts that the global rate of star formation should decline exponentially with a time constant $\tau_{0}$, and that the gas content should reach a finite asymptotic limit. Assuming that disks start off in a gaseous state, the implication is that gas content of a galaxy should be related to both the age of the disk and the current rate of star formation. The latter relationship is:

$$
\sigma_{\mathrm{g}} / \sigma_{\mathrm{T}}=\mathrm{f}_{2} \tau_{\mathrm{o}}\left(\partial \mathrm{M}_{*} / \partial \mathrm{t}\right) / \mathrm{M}_{\mathrm{T}}+\tau_{\mathrm{o}} / \tau_{1}
$$

where $\tau_{1}$ is a natural timescale called an 'Equipartition timescale', and is the time at which the pressure contribution of the older stellar population matches that of the younger.

The relationship (6.14) appears to give quite a good description of star-forming activity for all the galaxies in the Donas and Deharveng (1984) sample (see Figure 3 ).

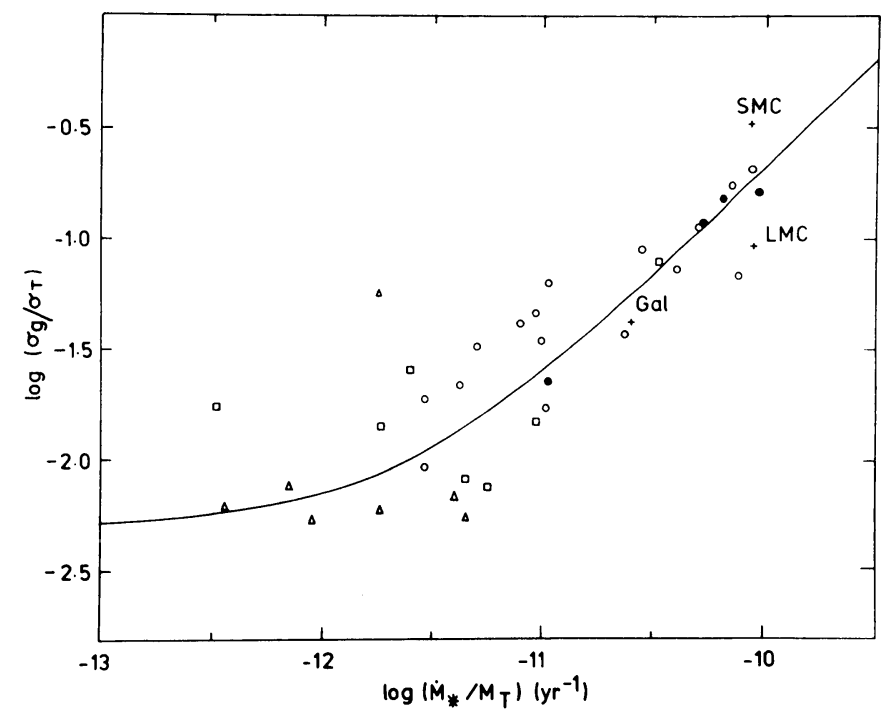

Figure 3: The specific rate of star-formation as a function of the specific surface density of gas. Symbols are as in Fig. 2, with Sa galaxies added as open triangles. The line represents the formal fit of Eqn. (6.14), with a solution $\mathrm{f}_{2} \tau_{0}=2$ Gyr and $\tau_{0} / \tau_{1}=0.005$.

This is somewhat remarkable, considering the range of absolute luminosities of galaxies included, the neglect of molecular gas and the relatively crude nature of the theory. 
The theory implies several observational consequences. Firstly, the gas depletion timescale, about $3 \mathrm{Gyr}$, is short compared with the Hubble timescale. Such short gas depletion timescales have been noted as a problem in the context of the Magellanic Clouds by Rocca-Volmerange, Lequeux and Maucherat-Joubert (1981). For other galaxies, this problem has been addressed in some detail by Kennicutt (1983) and Kennicutt and Kent (1983) who use H-Alpha emission as an index of star-formation. Timescales for gas depletion, defined observationally as the gas content divided by the rate of change of gas content, do range down to a few Gyr for gas rich systems, as equation (6.14) would predict.

Secondly, for all galaxies, the past rate of star-formation should have been hicher than the present one. This appears to be so, at least for the Galaxy and the Magellanic Clouds (see section 2), and this result could be checked observationally by measuring emission line equivalent widths and colours of galaxies at large look-back times.

Finally, gas content and the age of the disk should correlate in a one-to-one fashion.From the figures given in Table I, the local gas content of the Galaxy, and the global gas content of the LMC and SMC imply a disk age of $10 \mathrm{Gyr}, 7 \mathrm{Gyr}$ and $4 \mathrm{Gyr}$, respectively for these three systems. For the Magellanic Clouds, these numbers are in striking agreement with the mean age of the field stars in these systems; 4-7 Gyr for the LMC and 2-4 Gyr for the SMC (Butcher, 1977; Stryker 1981; Stryker and Butcher 1981; Hawkins and Brück, 1982,1984; Frogel and Blanco 1984). It is reasonable to hope, that, with the advent of ST, such studies might reveal a great deal about the temporal evolution of star formation rates for low-mass stars.

In conclusion,then, I hope that this review shows that an understanding of how the ISM and star formation react together is beginning to emerge. If this has been an optimistic overview, it reflects the author's view that, at last, the Magellanic Clouds are beginning to fufill their promise as local laboratories of the processes of star formation.

\section{REFERENCES}

Ardeberg, A., 1976. Astron. Astrophys. 46, 87

Azzopardi, M., 1982. in "La Structure du Petit Nuage de Magellan", Comptes rendues sur les Journées de Strasbourg; 4me. réunion, Obs. Strasbourg, p20

Azzopardi, M. and Vigneau, J., 1975. Astron. Astrophys. Suppl. Ser., 22, 285 Azzopardi, M. and Vigneau, J., 1977. Astron. Astrophys., 56, 151

Becker, S.A. and Iben, I.Jr., 1979. Astrophys.J., 232, 831

Becker, S.A. and Iben, I.Jr., 1980. Astrophys.J., 237, 111

Berkhuijsen, E.M., 1982. Astron. Astrophys. 112, 369

Braunsfurth, E. and Feitzinger, J.V., 1983. Astron. Astrophys., 127, 113

Bressan, A.G., Bertelli, G. and Chiosi, C. 1981. Astron. Astrophys., 102, 25

Bromage, G.E. and Nandy, K., 1983. Mon. Not. R. Ast. Soc., 204,29P 
Brück, M.T., 1975. Mon. Not. R. Ast. Soc., 173, 327

Bruhweiler, F.C., Gull, T.R., Kafatos, M. and Sofia, S., 1980. Astrophys. J. (Lett.), 238, L27

Butcher, H.R., 1977. Astrophys. J., 216, 373

Caulet, A., Deharveng, L., Georgelin, Y.M. and Georgelin, Y.P., 1982. Astron. Astrophys., 110, 185

Chiosi, C., Nasi, E. and Sreenivasan, S.R., 1978. Astron. Astrophys., 68, 467

Clegg, R.E.S., Lambert, D.L. and Tomkin, J., 1981. Astrophys. J., 250, 262

Cox, D.P., 1979. Astrophys. J., 234, 863

Cox, D.P., 1981. Astrophys. J., 245, 534

Cox, D.P. and Smith, B.W., 1974. Astrophys. J. (Lett.), 189, L105

de Boer, K.S., 1984. in "Structure and Evolution of the Magellanic Clouds", IAU Symposium \#108, Eds. S. van den Bergh and K.S. de Boer, Dordrecht: Reidel p 375

de Boer, K.S., Koorneef, J. and Savage, B.D., 1980. Astrophys.J., 236,769

de Boer, K.S. and Savage, B.D., 1980. Astrophys.J., 238,86

de Boer, K.S. and Nash, A.G., 1982. Astrophys.J. 255, 477; erratum 261, 747

de Vaucouleurs, G., 1955. Astron. J., 60, 219

de Vaucouleurs, G. and Freeman, K.C., 1973. Vistas in Astronomy, 14, 163

Dennefeld, M. and Tamman, G.A., 1980. Astron. Astrophys., 83, 275

Donas,, J. and Deharveng, J.M., 1984. Astron. Astrophys., 140, 325

Dopita, M.A., 1979. Astrophys. J. Suppl. Ser., 40, 455

Dopita, M.A., 1986. in "The Magellanic System", Ed. M.A.Dopita, Princeton U.P., in preparation

Dopita, M.A., Ford, H.C. Lawrence, C.J. and Webster, B.L., 1985. Astrophys. J., 296, 390

Dopita, M.A., Mathewson, D.S. and Ford, V.L., 1985. Astrophys. J., 296

Elmegreen, B.G. and Elmegreen, D.M., 1978. Astrophys. J., 220, 1051

Elmegreen, B.G. and Lada, C.J., 1977. Astrophys. J., 214, 725

Epchtein, N., Braz, M.A. and Sevre, F., 1984. Astron. Astrophys., 140, 67

Feitzinger, J.V. and Braunsfurth, E., 1984. Astron. Astrophys., 189, 104

Feitzinger, J.V., Glassgold, A.E., Gerola, H. and Seiden, P.E., 1981. Astron. Astrophys., 98, 371

Field, G.B. and Saslaw, W., 1965. Astrophys. J., 142, 583

Fitzpatrick, E.L. and Savage, B.D., 1984. Astrophys. J., 279, 578

Freeman, K.C., Illingworth, G. and Oemler, A. Jr., 1983. Astrophys. J., 272, 488

Frogel, J.A. and Blanco, V.M., 1983. Astrophys. J. (Letters), 274, L57

Fujimoto, M. and Murai, T., 1984. in "Structure and Evolution of the

Magellanic Clouds", IAU Symposium \#108, Eds. S. van den Bergh and

K.S. de Boer, Dordrecht: Reidel p 115.

Gatley, I., Hyland, A.R. and Jones, T.J., 1982. Mon. Not. R. Ast. Soc., 200,521

Gerola, H. and Seiden, P.E., 1978. Astrophys. J., 223, 129

Gerola, H., Seiden, P.E. and Schuman, L.S., 1980. Astrophys. J., 242, 517

Goudis, C. and Meaburn, J., 1978. Astron. Astrophys., 68, 169

Hamajima, K. and Tosa, M., 1975. Pupl. Ast. Soc. Japan, 27, 561

Hawkins, M.R.S. and Brück, M.T., 1982. Mon. Not. Roy. Ast. Soc., 198, 935

Hawkins, M.R.S. and Brück, M.T., 1984. in "Structure and Evolution of the

Magellanic Clouds", IAU Symposium \#108, Eds. S. van den Bergh and

K.S. de Boer, Dordrecht: Reidel p 101.

Hindman, J.V., 1967. Aust. J. Phys., 20, 147 
Huchra, J., 1977 Astrophys. J., 217, 928

Humphreys, R.M. and McElroy, D.B., 1984. Astrophys. J., 284,565

Inoue, H., Koyama, K. and Tanaka, Y., 1983. in"Supernova Remnants and their X-Ray Emission", Eds. J. Danziger and P. Gorenstein, Dordrecht:Reidel, p535

Israel, F.P., 1980. Astron. Astrophys., 90, 246

Isserstedt, J., 1984. Astron. Astrophys., 115, 97

Kaler, J.B., 1974. Astrophys. J. (Lett.), 188, L15

Koorneef, J. and Code, A.D., 1981. Astrophys. J., 247, 860

Larson, R.B and Tinsley B.M., 1978 Astrophys. J., 219, 46

Lequeux, J. 1980. in "Star Formation", 10th. Advanced Course of the Swiss Society of Astronomy and Astrophysics, Saas-Fee, Geneva Observatory

Lequeux, J., 1984. in"Structure and Evolution of the Magellanic Clouds", IAU Symposium \#108, Eds. S. van den Bergh and K.S. de Boer, Dordrecht: Reidel p67

Long, K.S., 1976. Doctoral Thesis, Calif. Inst. Tech., Pasadena, USA (ref.76-13,134)

Long, K.S., 1983. in"Supernova Remnants and their X-Ray Emission", Eds. J. Danziger and P. Gorenstein, Dordrecht:Reidel, p525

Long, K.S., Dopita, M.A. and Tuohy, I.R., 1982. Astrophys. J., 260, 202

Long, K.S., Helfand, D.J. and Grabelsky, D.A., 1981. Astrophys. J., 248, 925

Lucke, P.B. and Hodge, P.W., 1970. Astron. J., 75, 171

McCray, R. and Snow, T.P.Jr., 1979. Ann. Rev. Ast. Astrophys., 17, 213

McKee, C.F. and Ostriker, J.P., 1977. Astrophys. J., 218, 148

McKibben Nail, V. and Shapley, H., 1953. Proc. Natl. Acad. Sci. USA, 39, 358

Martin, N., Prévot, L., Rebeirot, E. and Rousseau, J., 1976. Astron. Astrophys. 51,31

Mathewson, D.S. and Ford, V.F., 1984. in"Structure and Evolution of the

Magellanic Clouds", IAU Symposium \#108, Eds. S. van den Bergh and

K.S. de Boer, Dordrecht: Reidel p125

Mathewson, D.S., Ford, V.L. and Visnanathan, N., 1985. Astrophys. J., (in press)

Meaburn, J., 1980. Mon. Not. Roy. Ast. Soc., 192, 365

Meaburn, J., 1981. in "Investigating the Universe", Astrophys. Space Sci. Library, 91,61 (Dordrecht:Reidel)

Maeder, A., 1981a. Astron. Astrophys. 99, 97

Maeder, A., 1981b. Astron. Astrophys. 102, 401

Maucherat-Joubert, M.,Lequeux,J. and Rocca-Volmerange, B., 1980.

Astron. Astrophys., 86, 299

Meatheringham, S. and Dopita, M.A. 1986. in prep.

Murai, T. and Fujimoto, M., 1980. Publ. Ast. Soc. Japan, 32, 581

Nomoto, K., 1984a. in "Stellar Nucleosynthesis", Eds. C.Chiosi and A.Renzini, Dordrecht:Reidel

Nomoto, K., 1984b. in "Stellar Nucleosynthesis", Eds. C.Chiosi and A.Renzini, Dordrecht:Reidel

Page, T. and Carruthers, G.R., 1981. Astrophys. J., 248, 906

Payne-Gaposhkin, C., 1972. in "L'Age des Etoiles", Ed. G. Cayrel de Strobel and A.M. Delplace. III, 1

Peimbert, M. and Torres-Peimbert, S, 1971. Astrophys. J., 168, 413

Renzini, A. and Voli, M., 1981. Astron. Astrophys., 94, 175 
Rocca-Volmerange, B., Lequeux, J.and Maucherat-Joubert, M., 1981. Astron. Astrophys., 104, 177

Rohlfs, K., Kreitschmann, J., Siegman, B.C. and Feitzinger, J.V., 1984. Astron. Astrophys., 137, 343

Sanduleak, N., 1968. Astron. J., 73, 246

Sanduleak, N., 1969a. Astron. J., 74, 877

Sanduleak, N., 1969b. Astron. J., 74, 47

Savage, B.D. and de Boer, K. S., 1979. Astrophys. J. (Lett.), 230, L77

Schmidt, M., 1959. Astrophys. J., 129, 243

Schmidt, Th., 1972. Astron. Astrophys., 16, 95

Schmidt-Kaler, Th., 1977. Astron. Astrophys., 54, 771

Searle, L., Sargent, W.L.W. and Bagnuolo, W., 1973. Astrophys. J., 179, 427

Seiden, P.E. and Gerola, H., 1979. Astrophys. J. 233, 56

Smith, L.F., Biermann, P. and Mezger, P. 1978. Astron. Astrophys., 66, 65

Songaila, A., 1981. Astrophys. J., 248, 945

Stryker, L.L., 1981. PhD. Thesis, Yale University.

Stryker, L.L. and Butcher, H.R., 1981. in "Astrophysical Parameters for

Globular Clusters", IAU Colloq. \#68, Ed. A.G.D.Philip and D.S. Hayes, Schenectady: L.Davis Press, p201

Sugimoto, D. and Nomoto, K., 1980. Space Sci. Review, 25, 155

Tosa M. and Hamajima, K., 1975.Pup. Ast. Soc. of Japan, 27, 501

Tinsley, B.M., 1972. Astron. Astrophys., 20, 383

Tuohy, I.R., Dopia, M.A., Mathewson, D.S., Long, K.S. and Helfand, D.J., 1982. Astrophys. J., 261, 473

van Genderen, A.M., 1969. Bull. Ast. Inst. Netherlands Suppl., 3, 221

Vangioni-Flam, E., Lequeux, J., Maucherat-Joubert, M.and Rocca-Volmerange, B., 1980. Astron. Astrophys. 90, 73

Weaver, T.A. and Woolsley, S.E., 1980. Ann. New York Acad. Sci., 366, 335

Weaver, T.A., Zimmerman, G.B. and Woolsley, S.E., 1978. Astrophys. J., 225, 1021

Westerlund, B.E., 1964a. Mon.Not.R.Ast. Soc., 27, 429

Westerlund, B.E., 1964b. "The Galaxy and the Magellanic Clouds"

IAU-URSI Symposium \#20, Eds. F.J. Kerr and A.W. Rodgers, Canberra;

Aust. Acad. Sci. p239

Westerlund, B.E. and Smith, L.F., 1964. Mon. Not. R. Ast. Soc., 128, 449

Westerlund, B.E. and Mathewson, D.S., 1966. Mon. Not. R. Ast. Soc., 131,371

Wilson, I.R.G., 1983, Ph.D. Thesis, Australian National University

Wood, P.R., Bessell, M.S. and Fox, M.W., 1983. Astrophys. J., 272, 99

Woosley, S.E. and Weaver, T.A., 1982. in "Essays in Nuclear Astrophysics", Eds. C.A. Barnes, D.D. Clayton and D.N. Schramm, Cambridge U.P.

YOUNG: I believe molecular gas was neglected? If so, is not the correlation of $\dot{M}$ vs. $\mathrm{M}_{\mathrm{T}} \sigma_{\mathrm{g}} / \sigma_{0}$ simply a correlation of $\dot{\mathrm{M}}$ vs. $\mathrm{M}_{\mathrm{T}}$ (since $\sigma_{\mathrm{HI}}$
tends to be the same in galaxies) and therefore that more massive galaxies are undergoing.more current epoch star formation? It would bt interesting to compare $\dot{\mathrm{M}}$ vs. $\sigma_{\mathrm{H}_{2}}$.

DOPITA: The molecular gas is indeed neglected in the Donas and Deharven (1984) sample. The correlation $\mathrm{M}_{\mathrm{T}} \sigma_{\mathrm{g}}$ vs. $\dot{M}$ refers to gas rich (low 
metallicity) objects only. I believe that molecular gas is important in producing the scatter in the $\sigma_{\mathrm{g}} / \sigma_{\mathrm{T}} \mathrm{vs} . \mathrm{M}_{*} / \mathrm{M}_{\mathrm{T}}$ correlation. It will also tend to change the slope (i.e. the derived value of $\tau_{0}$ ) and the intercept (the value of $\tau_{0} / \tau_{1}$ ). The correlation between $\dot{M}$ and $M_{T}$ shows a great deal more scatter, however.

SILK: Ionized gas goes up into the halo and cooler gas goes down in your model: should this effect show up for example, in observations of interstellar lines towards the LMC?

DOPITA: Yes, it should. The descending gas will have a relatively low area filling factor, however, and would be difficult to detect in absorption. It may be better seen in $\mathrm{HI}$.

PEIMBERT: There are models of spiral galaxy formation where the age of the disk is constant with galactocentric distance, according to your model the age of the disc decreases with galactocentric distance, is this result based only on the $\mathrm{M}_{\text {gas }} / \mathrm{M}_{\text {total }}$ ratio?

DOPITA: Yes.

PEIMBERT: Some estimates of the SMC M $\mathrm{gas}_{\mathrm{s}} / \mathrm{M}_{\text {total }}$ ratio are as high as 0.4 , according to your model is this value in contradiction with the average age of the stellar component of the SMC?

DOPITA: No, the estimates of the HI bound to the SMC is always lower than 0.4 . Earlier estimates were based on incomplete total photometry of the stellar parts. Dopita et al. (1985, Astrophys. J. October) have estimated the mass from the planetary nebula kinematics, and the gas fraction is near 0.25 . 